\title{
Publisher Correction: Nano-opto-electro-mechanical systems
}

Leonardo Midolo, Albert Schliesser and Andrea Fiore

Correction to: Nature Nanotechnology https://doi.org/10.1038/s41565-017-0039-1, published online 9 January 2018.

In the version of this Perspective originally published, in Fig. 1, in the green box labelled 'Mechanics', an erroneous grey rectangle was included; it has now been removed and the figure replaced in the online versions of the Perspective. 